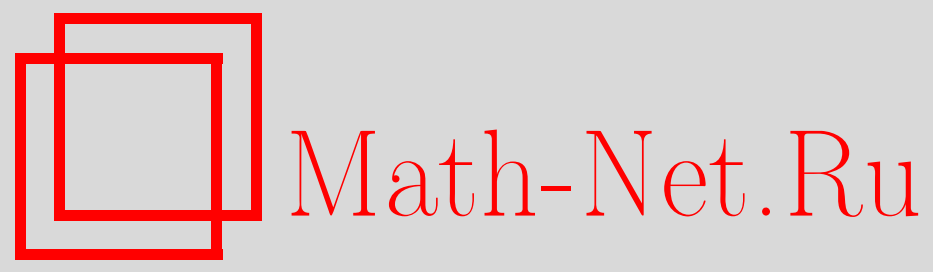

В. А. Колмыков, Эндоморфизмы функциональных графов, Дискрет. матем., 2006, том 18, выпуск 3, 115-119

DOI: https://doi.org/10.4213/dm64

Использование Общероссийского математического портала Math-Net.Ru подразумевает, что вы прочитали и согласны с пользовательским соглашением http://www . mathnet.ru/rus/agreement

Параметры загрузки:

IP : 54.198 .55 .26

26 апреля 2023 г., 10:17:56 
УДК 519.175

\title{
Эндоморфизмы функциональных графов
}

\author{
() 2006 г. $\quad$ В. А. Колмыков
}

\begin{abstract}
Функциональный граф - это орграф, описывающий действие функции на множестве. Эндоморфизмы таких орграфов представляют интерес в теории полугрупп: централизатор элемента полугруппы всех отображений множества в себя совпадает с множеством всех эндоморфизмов орграфа, соответствующего этому элементу. В статье рассматриваются функциональные графы со счетным множеством вершин. Введено понятие линеалов и вычислена мощность множества эндоморфизмов функциональных графов, не являющихся линеалами.
\end{abstract}

\section{1. Введение}

Рассмотрим отображение $g: M \rightarrow M$. Сколько существует отображений $f: M \rightarrow M$, для которых $f \circ g=g \circ f$ ?

На алгебраическом языке этот вопрос формулируется следующим образом. Симметрической полугруппой называется множество всех отображений $M \rightarrow M$ с операцией суперпозиции. Централизатором элемента $a$ полугруппы $S$ называется множество всех $b \in S$ таких, что $a b=b a$. Таким образом, нас интересует, какова мощность централизатора произвольного элемента симметрической полугруппы.

Несмотря на простоту такого вопроса, ответа на него в настоящее время нет. Причина этого, вероятно, в том, что вопрос находится на стыке алгебры и теории множеств, а отвечать на него удобно на языке теории графов.

Действие отображения $g: M \rightarrow M$ на множестве $M$ можно представить орграфом $O_{g}$ со множеством вершин $M$ и множеством стрелок $E=\{(x, g(x))\}_{x \in M} \subseteq M^{2}$. Централизатор элемента $g$ симметрической полугруппы совпадает с множеством всех эндоморфизмов орграфа $O_{g}$.

Идея исследовать централизатор как множество эндоморфизмов некоторого объекта принадлежит классу простых алгебраических идей. Однако для ее реализации необходимо осуществлять нетривиальные теоретико-графовые рассмотрения. Орграф $O_{g}$ - это так называемый функциональный граф. Эндоморфизмы функциональных графов изучались, например, в [1-3] с алгебраической точки зрения и с дополнительными условиями. В [4] на теоретико-графовом языке вычислена мощность централизатора инъекщии или сюръекции, действующей на счетном множестве.

В настоящей статье мы рассматриваем функциональные графы со счетным множеством вершин. Мы вводим понятие линеалов и доказываем, что мощность множества эндоморфизмов счетных функциональных графов, не являющихся линеалами, равна $\aleph_{0}^{k \tau}$, где $k$ - число компонент, а $\tau$ - ветвление орграфа.

Вопрос о мощности множества эндоморфизмов линеала остается нерешенной проблемой. 


\section{2. Формулировка результата}

Орграф - это пара $(V, E)$, где $E \subseteq V^{2}$. Элемент $\overrightarrow{u v}=(u, v) \in E$ называется стрелкой с началом $u$ и конщом $v$. Орграф со счетным множеством вершин называется счетным.

Эндоморфизм орграфа - это отображение множества его вершин в себя $v \mapsto \tilde{v}$ такое, что наличие стрелки $\overrightarrow{u v}$ влечет наличие стрелки $\overrightarrow{\tilde{u} v}$. Множество всех эндоморфизмов орграфа $G$ обозначается $\operatorname{End}(G)$.

Функциональный граф - это орграф, в котором из каждой вершины выходит ровно одна стрелка (на число входящих стрелок ограничений не накладывается, стрелки-петли допускаются).

Напомним, что две вершины слабо связаны, если они связаны в неориентированном графе, получающемся заменой стрелок ребрами. Мощность множества компонент слабой связности орграфа $G$ обозначается $k(G)$.

Степенью вершины орграфа называется сумма мощности множества входящих в нее стрелок и мощности множества выходящих из нее стрелок (стрелка-петля принадлежит обоим множествам). Узел - это вершина степени, не меньшей 3. Ветвлением $\tau(G)$ орграфа $G$ назовем сумму степеней его узлов; если в $G$ нет узлов, то положим $\tau(G)=2$.

Если все ребра корневого дерева ориентировать по направлению к корню, то полученное корневое ордерево будем называть ориентированным к корню. Одновершинное корневое дерево $P_{1}$ удобно считать ориентированным к корню. Множество всевозможных конечных корневых ордеревьев, ориентированных к корню, обозначим $\dot{\mathfrak{T}}_{\mathrm{fin}}$. В каждой конечной $n$-вершинной цепи $P_{n}$, где $n \geqslant 2$, в качестве корня возьмем концевую вершину. Каждую такую корневую цепь ориентируем к корню. Множество, состоящее из полученных корневых орцепей и $P_{1}$, обозначим $\dot{P}$.

Пусть $\alpha=\left\{\alpha_{i}\right\}_{i \in \mathrm{N}}$ - последовательность с членами из множества $\dot{\mathscr{T}}_{\text {fin }}$. Рассмотрим орграф $\vec{N}$, для которого $V=\left\{v_{i}\right\}_{i \in \mathbf{N}}, E=\left\{\overrightarrow{v_{i} v_{i+1}}\right\}_{i \in \mathbf{N}}$. Через $\vec{N}(\alpha)$ обозначим орграф, получающийся отождествлением корня орграфа $\alpha_{i}$ и вершины $v_{i}$ для любого $i \in \mathbf{N}$. Последовательность $\alpha$ назовем почти $\dot{\mathscr{P}}$-последовательностью, если выполнены два условия:

(1) для всех достаточно больших $i$ справедливо включение $\alpha_{i} \in \dot{P}$,

(2) множество индексов $i$, для которых $\alpha_{i} \neq P_{1}$, бесконечно.

Пусть $K$ - либо пустой орграф, либо функщиональный граф конечного ветвления, имеющий конечное число компонент (то есть $k \tau<\aleph_{0}$ ), а $\alpha^{(1)}, \ldots, \alpha^{(s)}-$ набор почти $\dot{\mathscr{P}}$-последовательностей.

Линеалом назовем орграф

$$
K \cup \vec{N}\left(\alpha^{(1)}\right) \cup \ldots \cup \vec{N}\left(\alpha^{(s)}\right) .
$$

Теорема 1. Если $G$ - счетный функциональный граф, не являющийся линеалом, то

$$
|\operatorname{End}(G)|=\aleph_{0}^{k(G) \tau(G)} .
$$

\section{3. Доказательство теоремы}

Утверждение теоремы вытекает из нижеследующих лемм 1, 2 и 4. Первые две леммы доказаны в [4]. 
Лемма 1. Пусть $G-$ счетный функциональный граф и $k(G)=\aleph_{0}$. Тогда

$$
|\operatorname{End}(G)|=\aleph_{0}^{\aleph_{0}} \text {. }
$$

Лемма 2. Если $G-$ счетный функциональный граф и $k(G) \tau(G)<\aleph_{0}$, то

$$
|\operatorname{End}(G)|=\aleph_{0} \text {. }
$$

Для доказательства лемм 3-4 введем следующие обозначения и понятия.

Множество всех вершин орграфа $G$ обозначается $V(G)$. Пусть $G-$ функциональный граф. Если $v \in V(G)$, то через $v^{+}$обозначается вершина, являющаяся концом стрелки, выходящей из $v$.

Нитью назовем нумерованное подмножество $\left\{u_{i}\right\}_{i \in Z}$ множества $V(G)$ такое, что $\left(u_{i}\right)^{+}=u_{i+1}$ для любого $i$. Пусть $l \in \mathbf{N}, I_{l}=[-l ; 0] \cap Z$. Конечным хвостом (длины $l$ ) вершины $v_{0}$ назовем нумерованное подмножество $\left\{v_{i}\right\}_{i \in I_{l}} \subseteq V(G)$ такое, что $\left(v_{i}\right)^{+}=v_{i+1}$ для любого ненулевого индекса $i \in I_{l}$. Пусть $I$ - множество всех неположительных целых чисел. Бесконечным хвостом вершины $v_{0}$ назовем нумерованное подмножество $\left\{v_{i}\right\}_{i \in I} \subseteq V(G)$ такое, что $\left(v_{i}\right)^{+}=v_{i+1}$ для любого ненулевого индекса $i \in I$.

Множество всех вершин, принадлежащих какому-либо хвосту вершины $u$, обозначим $T(u)$. Хвост $\left\{u_{i}\right\}_{i \in I}$ назовем простым, если ни одна из вершин, имеющих отрицательный индекс, не принадлежит контуру орграфа (если таковой имеется). Пусть $\left\{u_{i}\right\}_{i \in I}-$ простой хвост. Расстоянием $r\left(u_{i}, u_{j}\right)$ от $u_{i}$ до $u_{j}$ назовем $|i-j|$.

Пусть зафиксировано несколько простых хвостов вершины $v$. Через $\tilde{T}(v)$ обозначим множество всех вершин, входящих хотя бы в один из зафиксированных хвостов. Пусть $\left\{u_{i}\right\}_{i \in I}$ - некоторый хвост. Будем говорить, что отображение $g: V(G) \rightarrow V(G)$ схлопывает $\widetilde{T}(v)$ в $\left\{u_{i}\right\}_{i \in I}$, если $g$ переводит вершину $w \in \tilde{T}(v)$ в $u_{-r}(w, v)$. При этом, если $M \subseteq \widetilde{T}(v)$, то будем говорить, что $M$ схлопывается в $\left\{u_{i}\right\}_{i \in I}$.

Орграф, в котором отмечена одна вершина, называется корневым, а отмеченная вершина - корнем. Эндоморфизмом корневого орграфа называется всякий эндоморфизм орграфа, переводящий корень в корень. Включение $G \subseteq G^{\prime}$ корневых орграфов $G=(V, E)$ и $G^{\prime}=\left(V^{\prime}, E^{\prime}\right)$ означает включения $V \subseteq V^{\prime}, E \subseteq E^{\prime}$ и совпадение их корней.

Пусть $\overleftarrow{N}$ - корневое ордерево, для которого $V=\left\{v_{i}\right\}_{i \in \mathbf{N}}, E=\left\{\overrightarrow{v_{i} v_{i-1}}\right\}_{i \geqslant 2}$, вершина $v_{1}$ является корнем.

Лемма 3. Пусть корневое ордерево $G$ ориентировано к корню и не принадлежит множеству $\dot{P} \cup\{\overleftarrow{N}\}$. Тогда $\operatorname{End}(G)$ содержит не менее двух элементов.

Доказательство. Нам надо построить некоторый нетождественный эндоморфизм. Корень орграфа $G$ обозначим $v$.

Пусть среди вершин из $V(G) \backslash\{v\}$ есть вершина $u$ степени один. Через $w$ обозначим ближайшую к $u$ вершину такую, что она есть либо узел, либо корень. Хвост $\{u, \ldots, w\}$ обозначим $V_{1}$ и положим $V_{2}=T(w) \backslash V_{1}$. Если существует вершина $u^{\prime} \in V_{2}$ такая, что $r\left(u^{\prime}, w\right) \geqslant r(u, w)$, то искомый эндоморфизм определим как тождественный на $V_{2}$, а хвост $V_{1}$ схлопывается в хвост $\left\{u^{\prime}, \ldots, w\right\}$. Если для любой вершины $u^{\prime} \in V_{2}$ справедливо неравенство $r\left(u^{\prime}, w\right)<r(u, w)$, то искомый эндоморфизм определим как тождественный вне $V_{2}$, а $V_{2}$ схлопывается в $V_{1}$.

Пусть все вершины из $V(G) \backslash\{v\}$ имеют степень, не меньшую 2. Тогда $\overleftarrow{N} \subset G$. Искомый эндоморфизм определим как тождественный на $V(\overleftarrow{N})$, а остальные вершины схлопываются в $V(\overleftarrow{N})$. 
Лемма 4. Пусть $G$ - счетный функциональный граф и $k(G)<\aleph_{0}$. Если $\tau(G)=\aleph_{0} u G$ не является линеалом, то

$$
|\operatorname{End}(G)|=\aleph_{0}^{\aleph_{0}}
$$

Доказательство. Очевидно, что для всякого счетного орграфа $|\operatorname{End}(G)| \leqslant \aleph_{0}^{\boldsymbol{\aleph}_{0}}$.

Ветвлением $\tau(v)$ вершины $v$ назовем сумму степеней узлов из $T(v)$. Рассмотрим три возможных случая.

Случай 1. Пусть в $G$ имеется вершина $u$ бесконечной степени. Пусть $\left\{u_{i}\right\}_{i \in \mathrm{N}}-$ множество вершин таких, что ни одна из них не принадлежит контуру и из каждой из этих вершин направлена стрелка в $u$. Рассмотрим орграф, порожденный вершинами $\{u\} \cup T\left(u_{2 i-1}\right) \cup T\left(u_{2 i}\right)$. Если в нем присутствует стрелка-петля $\vec{u}$, то удалим ее (саму же вершину $u$ сохраним). В качестве корня выберем вершину $u$. Полученное корневое ордерево ориентировано к корню. Обозначим его $G_{i}$. Для всякого $i \in \mathbf{N}$ множество $\operatorname{End}\left(G_{i}\right)$ содержит (согласно лемме 3 ) не менее двух элементов. Поэтому $\operatorname{End}(G)$ содержит не менее $2^{\boldsymbol{\aleph}_{0}}$ элементов.

Случай 2. Пусть в $G$ нет вершин бесконечной степени, но есть вершина бесконечного ветвления. Выберем вершину $v_{0}$ бесконечного ветвления, не принадлежащую контуру. Вершины $v_{-1}, v_{-2}, \ldots$ бесконечного ветвления выберем индуктивно: $v_{i}^{+}=v_{i+1}$. Определим бесконечный хвост $T_{*}\left(v_{i}\right)=\left\{u_{n}\right\}_{n=0,-1,-2, \ldots}$, где $u_{n}=v_{i+n}$. Через $T^{*}\left(v_{i}\right)$ обозначим объединение всех хвостов вершины $v_{i}$, не содержащих вершину $v_{i-1}$. Через $M$ обозначим множество всех индексов $i$ таких, что вершина $v_{i} \in T_{*}\left(v_{0}\right)$ является узлом. Множество $M$ бесконечно (иначе для достаточно больших $i$ ветвление $v_{i}$ равнялось бы двум). Для всякого $P \subseteq M$ определим $\varphi_{P} \in \operatorname{End}(G)$ следующим образом. Для всякого $j \in P$ отображение $\varphi_{P}$ схлопывает $T^{*}\left(v_{j}\right)$ в $T_{*}\left(v_{j}\right)$. На вершинах, не принадлежаших объединению $\bigcup_{j \in P} T^{*}\left(v_{j}\right)$, отображение $\varphi_{P}$ действует как тождественное. Множество отображений вида $\varphi_{P}$ имеет мощность $2^{\left.\aleph_{1}\right)}$.

Случай 3. Пусть все вершины $G$ имеют конечное ветвление.

В $G$ есть компонента $H$ бесконечного ветвления, не являющаяся линеалом. Покажем, что в $H$ нет контура. Предположим, что контур есть. Множество его вершин обозначим $V_{1}$. Через $V_{2}$ обозначим множество всех вершин $v \in V(H) \backslash V_{1}$ таких, что $v^{+} \in V_{1}$. Множество $V_{2}$ конечно. Ветвление $\tau(H)$ равно сумме ветвлений вершин из $V_{2}$ плюс некоторое конечное кардинальное число. Выходит, что $\tau(H)<\aleph_{0}$, получаем противоречие.

Выберем в $H$ произвольным образом вершину $v_{0}$. Для натуральных $i$ положим $v_{i}=\left(v_{i-1}\right)^{+}$; через $T^{*}\left(v_{i}\right)$ обозначим объединение всех хвостов вершины $v_{i}$, не содержащих вершину $v_{i-1}$. Рассмотрим орграф, порожденный вершинами из $T^{*}\left(v_{i}\right)$. Отметим в нем вершину $v_{i}$. Полученное корневое ордерево обозначим $H_{i}$. Через $M$ обозначим множество всех индексов, для которых $H_{i} \neq P_{1}$. Так как каждое $H_{i}$ имеет конечное ветвление, а ветвление $H$ бесконечно, множество $M$ бесконечно.

Пусть $H_{i}$ конечно для любого $i \in M$. Через $M^{\prime}$ обозначим множество всех $i \in M$, для которых $H_{i} \notin \dot{\mathscr{P}}$. Так как $H-$ не линеал, множество $M^{\prime}$ бесконечно. Для всякого $i \in M^{\prime}$ множество $\operatorname{End}\left(H_{i}\right)$ содержит (согласно лемме 3) не менее двух элементов. Поэтому $\operatorname{End}(H)$ содержит не менее $2^{\boldsymbol{N}_{0}}$ элементов.

Пусть $H_{s}$ бесконечно для некоторого $s$. Тогда $\overleftarrow{N} \subseteq H_{s}$. Если $i>s$, то определим бесконечный хвост $T_{*}\left(v_{i}\right)=\left\{u_{n}\right\}_{n=0,-1, \ldots}$, где $u_{n}$ - вершина из $\left\{v_{m}\right\}_{s+1 \leqslant m \leqslant i} \cup V(\overleftarrow{N})$, находящаяся на расстоянии $|n|$ от $v_{i}$. Для всякого $P \subseteq M \cap(s ;+\infty)$ определим $\varphi_{P} \in \operatorname{End}(G)$ 
следующим образом. Для всякого $j \in P$ отображение $\varphi_{P}$ схлопывает $T^{*}\left(v_{j}\right)$ в $T_{*}\left(v_{j}\right)$. На вершинах, не принадлежащих объединению $\bigcup_{j \in P} T^{*}\left(v_{j}\right)$, отображение $\varphi_{P}$ действует как тождественное. Множество отображений вида $\varphi_{P}$ имеет мощность $2^{\aleph_{0}}$.

Следующие вопросы остаются открытыми.

1. Какова мощность множества эндоморфизмов линеала?

2. Последовательности $\alpha$ и $\beta$ назовем эквивалентными, если существуют числа $m$ и $n$ такие, что $\alpha_{m+i}=\beta_{n+i}$ для любого $i \in \mathbf{N}$. Ростком назовем класс эквивалентных последовательностей. Верно ли, что мощность множества эндоморфизмов линеала $K \cup \vec{N}\left(\alpha^{(1)} \cup \ldots \cup \vec{N}\left(\alpha^{(s)}\right.\right.$ есть функция ростков последовательностей $\alpha^{(1)}, \ldots, \alpha^{(4)}$ ?

\section{Список литературы}

1. Varlet J. C., Endomorphisms and fully invariant congruences in unary algebras $\langle A ; \Gamma\rangle$. Bull. Soc. Roy. Sci. Liege (1970) 39, №11-12, 575-589.

2. Скорняков Л. А., Групповые унары. Вестник МГУ. Матем. Мех. (1977), №5, 65-69.

3. Бочкин А. М., Унары с сепаративным моноидом эндоморфизмов. Изв. вузов, сер. матем. (1983), №5, 71-74.

4. Колмыков В. А., О соотношении коммутативности в симметрической полугруппе. Сиб. матем. журн. (2004), 45, №5, 1130-1135.

Статья поступила 31.11.2004. 